\title{
Diagnostic Efficacy of CXR Compared to Chest CT in Preoperative Staging of Patients with Colorectal Cancer
}

\author{
Sahar Rabani Khorasgani ${ }^{1,{ }^{*}}$, Farhad Niaghi ${ }^{1}$ and Pooneh Dehghan ${ }^{1}$ \\ ${ }^{1}$ Department of Radiology, Taleghani Hospital Research Development Committee, School of Medicine, Shahid Beheshti University of Medical Sciences, Tehran, Iran \\ "Corresponding author: Department of Radiology, Taleghani Hospital Research Development Committee, School of Medicine, Shahid Beheshti University of Medical Sciences, \\ Tehran, Iran. Email: rabani1369@yahoo.com
}

Received 2018 August 01; Revised 2019 March 19; Accepted 2019 April 07.

\begin{abstract}
Background: The high prevalence of colorectal cancer (CRC) and its increasing incidence can be a major problem in cancer management. Preoperative staging plays a significant role in the therapeutic process, especially in CRC which has high risk for lung metastases.

Objectives: This study aimed to evaluate accuracy of chest X-ray (CXR) in comparison with chest CT(CCT) for diagnosing lung metastases in patients with CRC.

Patients and Methods: A cross sectional study was conducted of 377 patients with newly diagnosed CRC which were evaluated with CXR and CCT.

Results: CCT revealed normal/benign findings in 285 (75.6\%), indeterminate nodules in 28 (17\%) and suspicious nodules in 64 (17\%) patients. Although CXR did not reveal any pulmonary metastatic lesions in of the 23(7\%) patients, the measure of agreement between CXR and CCT was good $(0.64, \mathrm{P}<0.01)$ and CCT changed TNM staging in only eight $(2 \%)$ patients. On logistic regression analysis, liver metastasis could increase the risk of pulmonary metastasis $(\mathrm{P}<0.01)$.

Conclusion: The study suggested CCT for preoperative staging in CRC patients, especially at stages 3 and 4 in TNM staging and for liver metastases.
\end{abstract}

Keywords: Colorectal Cancer (CRC), Chest CT (CCT), Chest X-Ray (CXR), Lung Metastases, Indeterminate Lung Lesions

\section{Background}

The high prevalence of colorectal cancer (CRC) and its increasing incidence can be a major problem in cancer management (1). The incidence of rectal cancer is estimated to be $35 \%$ of total CRC incidence in the European Union (2). The lung is the most common extrahepatic site of distant metastasis from CRC with an incidence of $10 \%$ $(3,4)$. Lower rectal tumors have a higher incidence of lung metastasis owing to systemic venous drainage of this portion as opposed to the venous drainage of the colon through the portal system $(3,5)$. It has been hypothesized that, in the absence of hepatic metastasis, it is uncommon for colonic tumors to spread to the lung (6). When pulmonary metastasis is diagnosed, a patient no longer undergoes unnecessary colorectal surgery and is given alternative treatment (5). The precise staging like the tumor, node, metastasis (TNM) staging system is important for deciding upon CRC treatment protocol $(2,7)$ and lung evaluation is helpful in determining metastasis in staging. The low incidence of pulmonary metastasis, the cost of the CT scan, exposure to radiation and uncertainty of treatment due to the prevalence of unspecified lesions limits the clinical value of routine preoperative chest CT (CCT) (7).

\section{Objectives}

The current study was undertaken to compare the efficacy of chest X-ray (CXR) and CCT in preoperative staging of colon and rectal cancer.

\section{Patients and Methods}

\subsection{Patients and Imaging Studies}

In a cross-sectional study, 377 patients with new histopathological diagnoses of CRC referring to Taleghani hospital, a tertiary care referral center for gastrointestinal diseases in Tehran, Iran, were included for preoperative screening between 2014 and 2017. CRC cases with To values were excluded. The patients underwent both CXR and CCT 
without contrast. The data on liver metastasis and paraaortic lymphadenopathy were gathered by abdominal CT scan with contrast. PA digital radiography was taken in the upright position. A CT scan of the abdomen was performed on a 16-slice scanner (Toshiba Aquillion) after intravenous contrast injection (visipaque $320 ; 90 \mathrm{~mL} ; 3 \mathrm{~mL} / \mathrm{sec}$ ) in the portal venous phase with a slice thickness of $1 \mathrm{~mm}, 120$ $\mathrm{kVp}, 250 \mathrm{mAs}$ and reconstruction of $0.8 \mathrm{~mm}$. The images were revised by a radiologist with 15 years of experience in thorax imaging. The CXR findings were classified as nor$\mathrm{mal} /$ benign or suspicious and the CCT findings were classified as normal/benign, indeterminate or suspicious nodules. The interval of CXR and CCT reporting was 10 days to prevent recall bias. Patients with suspicious nodules in CCT underwent diagnostic procedures such as a CT-guided biopsy, bronchoscopy and thoracotomy depending on the location of lesions and status of the patients or were followed with chemotherapy treatment.

\subsection{Ethical Statement}

All patients were followed up with a detailed description of the purpose of the research and written consent was obtained. Patient names were not included in the study and coding was used to classify the information. The benefits of participation in the plan were to undertake an important step in the staging of the tumor before surgery which has a significant effect on the treatment process. The cost of participation in the scheme was free. Possible complications included exposure to radioactive radiation, sensitivity and nephropathy by contrast media. The likelihood of contrast-induced nephropathy is about $2 \%$ and can be prevented by evaluation of glomerular filtration rate (GFR) and hydration of patients with borderline GFR (8).

\subsection{Classification of Lesions}

A benign nodule was defined as a small uncalcified nodule showing no changes when compared with the prior CT (9). An indeterminate nodule is defined as a nodule that shows no calcification and was less than $5 \mathrm{~mm}$ in size when a prior CCT is not available (10). A suspicious nodule is defined as having a smooth margin, monogonal shape and diffuse scattered distribution (11) (Figure 1).

\subsection{Statistical Analysis}

Descriptive statistics were used to summarize the variables. The variables recorded included age, gender, CXR and CCT findings, paraaortic lymphadenopathy and liver metastasis. Linear regression was used to correlate the liver metastases, paraaortic lymphadenopathy and pulmonary metastases. IBM SPSS Statistics for Windows version 24.0 (IBM Corp. Released 2016, Armonk, NY: IBM Corp) was used for analysis. A P value of less than 0.05 was considered statistically significant. The data was expressed as mean and percentile. The Cohen kappa test was used for agreement between CXR and CCT. The McNemar test was used to compare the sensitivity, specificity and accuracy of the chest CT group and the CXR group in comparison with pathology.

\section{Results}

\subsection{CCT and CXR}

Of the 377 patients enrolled in this study, 59.7\% of patients were male and $40.3 \%$ of patients were female. Patients with colon cancer were more prevalent (65\% vs. $35 \%$ ) than those with rectal cancer and had higher staging according to TNM scoring $(\mathrm{P}<0.01)$. The distribution of TNM staging in colorectal patients was $3(8 \%)$ patients for stage 1, 94 (24.9\%) patients for stage 2, 91 (24.1\%) patients for stage 3 and $189(50.1 \%)$ for stage 4 .

Their demographic characteristics are shown in Table 1. In the CXR group, 329 (87.35\%) of patients were diagnosed as having normal or benign findings and 48 (12.7\%) of patients showed suspicious nodules. In the chest CT group, 285 (76\%) of patients were normal/benign nodules, 28 (7\%) had indeterminate nodules and 64 (17\%) had suspicious nodules (Figure 2). Patients who had pulmonary metastases on the pre-operative chest CT underwent diagnosis (Table 2).

\subsection{Comparison Between CCT and CXR}

The measure of agreement between CXR and CCT was $\operatorname{good}(0.64, \mathrm{P}<0.01)$ (95\% confidence interval $(\mathrm{CI})=0.58$ 0.69). Detecting malignant lesions by CCT was higher than CXR since the sensitivity of the chest CT group was $96.2 \%$ and that of the CXR group was $76.9 \%$ in comparison with pathology. The accuracy was $96.2 \%$ in the chest CT group and $85.9 \%$ in the CXR group. There was no difference in specificity between groups (96.2\% vs. 95\%). There was moderate agreement between TNM staging and rate of malignant nodules in the CCT group $(0.50, \mathrm{P}<0.01)(95 \% \mathrm{CI}=$ $0.492-0.508)$.

Among these patients, 21 (5.5\%) had isolated pulmonary metastases, 40 (10.6\%) had simultaneous hepatic metastases and 35 (9.2\%) had extra pulmonary and hepatic seeding. Significant discrepancy was detected in the incidence of isolated hepatic metastasis between the colon 

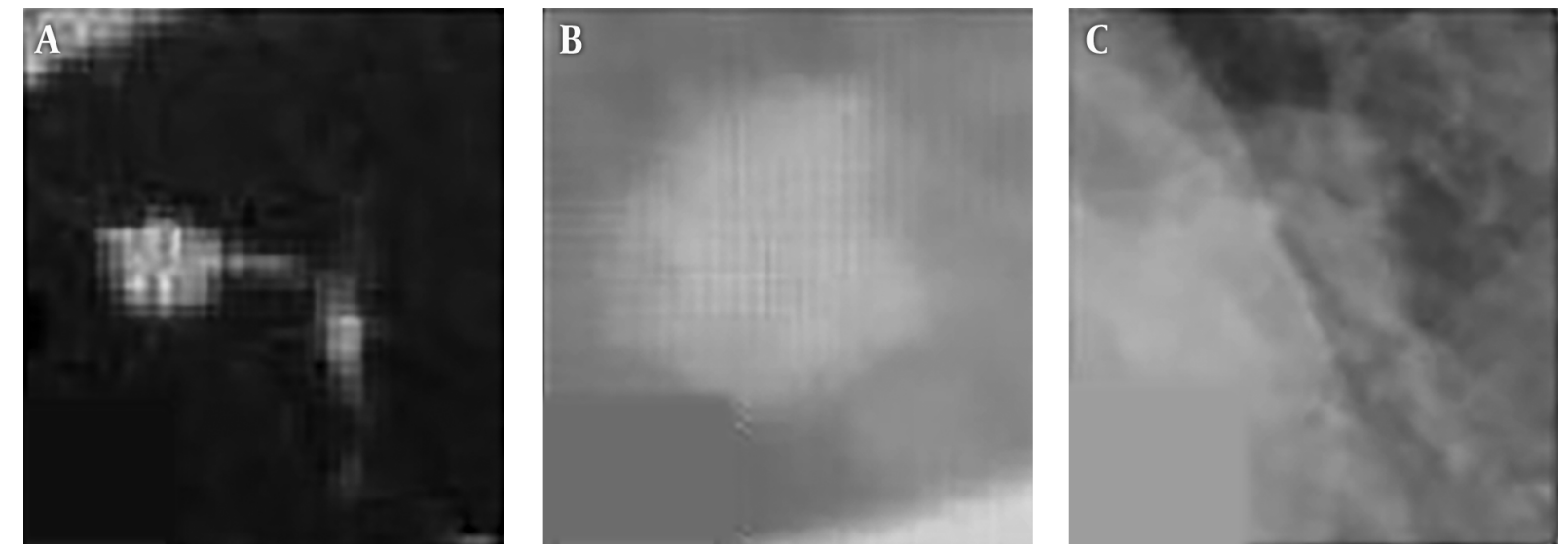

Figure 1. Classification of the radiologic findings. A, Indeterminate nodule in CCT; B, Suspicious nodule in CCT; C, Suspicious nodule in CXR(CXR, chest X-ray; CCT, chest CT)

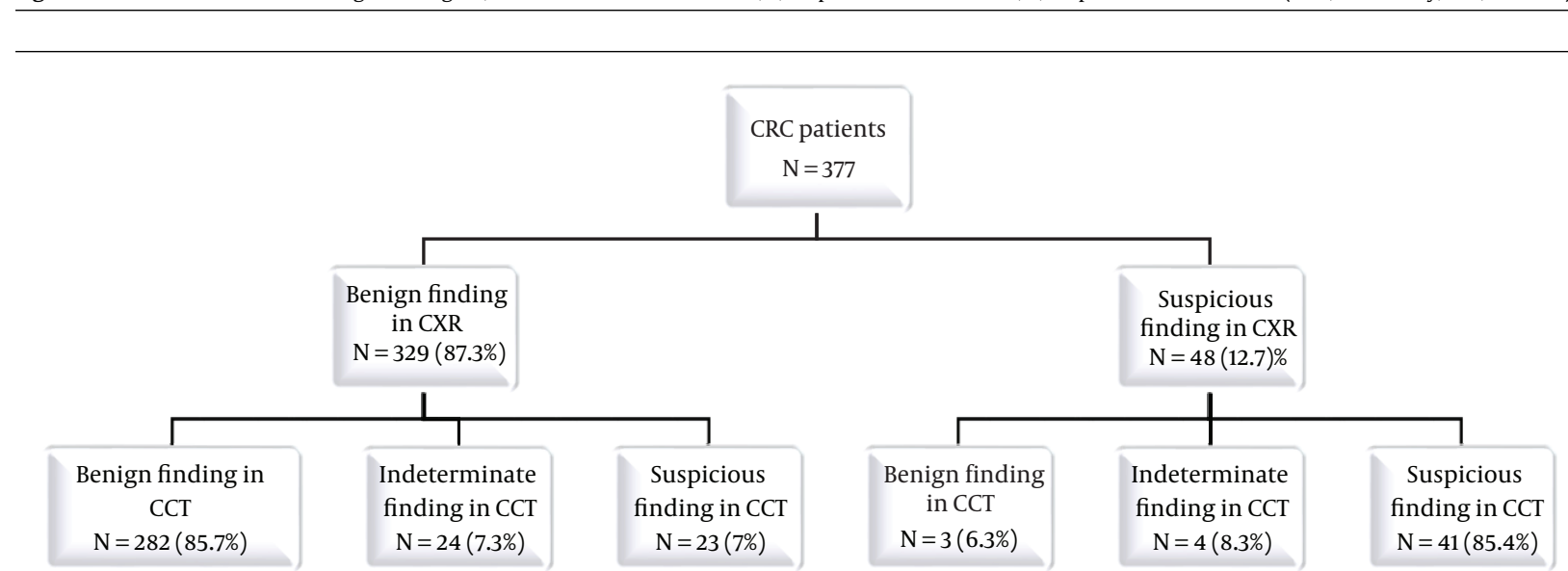

Figure 2. Characteristics of CXR and CCT in patients with CRC (CXR, chest X-ray; CRC, colorectal cancer; CCT, chest CT)

and rectal group $(\mathrm{P}<0.01)$. On logistic regression analysis, liver metastasis could increase the risk of pulmonary metastasis in colon $(\mathrm{P}=0.02)$ and rectal cancer $(\mathrm{P}<0.01)$, but there was no correlation between paraaortic lymphadenopathy and lung involvement in colon $(\mathrm{P}=0.69)$ and rectal cancer $(\mathrm{P}=0.90)$.

\section{Discussion}

Most guidelines agree that the chest should be imaged for staging of CRC, but there is disagreement about the exact type of imaging modality (11). The National Comprehensive Cancer Network has recommended CT rather than plain film radiographic imaging of the chest (12). The main reason for the use of CT for staging is early diagnosis of pulmonary metastases with high sensitivity of $51 \%$ to $73 \%$ and specificities of up to $74 \%$, which increases the rate of resection of pulmonary metastasis $(4,11)$. CCT is competent for identifying nodules of 2 - $3 \mathrm{~mm}$, whereas CXR identifies lesions 5-10 $\mathrm{mm}$ in size (13). Early detection and resection of pulmonary metastases from CRCs may result in 5-year survival rates above $50 \%$ in carefully selected patients (7).

Some studies have argued that routine CCT should not be a part of preoperative staging because pulmonary metastasis has a low incidence and CCT occasionally influences the treatment plan and has a risk of radiation exposure $(7,13,14)$. In addition, an indeterminate nodule can change pulmonary staging, but does significantly effect on patient's clinical course (11). The low positive yield and low positive predictive value of CCT when healing with a negative CXR brings the usefulness of routine CCT under question (15). In this research, although CXR did not reveal any pulmonary metastatic lesions in 23 (7\%) patients, the mea- 


\begin{tabular}{|c|c|}
\hline Variable & No. (\%) \\
\hline \multicolumn{2}{|l|}{ Age } \\
\hline Mean & 57 \\
\hline Median & 58 \\
\hline Range & $17-91$ \\
\hline \multicolumn{2}{|l|}{ Gender } \\
\hline Male: Female & $1.48: 1.0$ \\
\hline \multicolumn{2}{|l|}{ Location } \\
\hline Rectum & $132(35)$ \\
\hline Colon & $245(65)$ \\
\hline \multicolumn{2}{|c|}{ AJCC stage based on TNM } \\
\hline 1 & $3(0.8)$ \\
\hline 2 & $94(24.9)$ \\
\hline 3 & $91(24.1)$ \\
\hline 4 & $189(50.1)$ \\
\hline \multicolumn{2}{|l|}{ Liver metastases } \\
\hline Yes & $139(36.9)$ \\
\hline No & $238(63.1)$ \\
\hline \multicolumn{2}{|c|}{ Paraaortic adenopathy } \\
\hline Yes & $73(19.4)$ \\
\hline No & $304(80.6)$ \\
\hline
\end{tabular}

Abbreviations: AJCC, American joint committee on cancer; CRC, colorectal cancer; TNM, tumor, node, metastasis

Table 2. Additional Diagnostic Tests for Indeterminate Lesions

\begin{tabular}{lc}
\hline & Patients $(\mathbf{N}=\mathbf{5 9})$ \\
\hline CT guided biopsy & 17 \\
Bronchoscopy & 7 \\
\hline Thoracotomy & 3 \\
\hline Routinely followed-up with chemotherapy & 25 \\
\hline Expired before diagnostic procedure & 7 \\
\hline
\end{tabular}

sure of agreement between CXR and CCT was good (0.64, $\mathrm{P}<0.01)$ and CCT changed TNM staging in only eight $(2 \%)$ patients.

One main problem of staging with chest CTs is in the finding of indeterminate lesions which have a low risk of malignancy $(10 \%-20 \%)$ and are usually difficult to determine $(7,11)$. Small nodules are often seen on chest CTs and are usually benign (7). The number of indeterminate lesions in this study was $17 \%$, while most studies estimate it about $20 \%-30 \%$. Heterogeneity in studies and the lack of agreement in the definition of ILL confirms this (4). Radiologist experience and improvements in scanning technolo- gies will increase the identifying such lesions (16). A small proportion of indeterminate lung lesions develop into definite metastases $(<30 \%)$, mostly in node positive disease. This is a clue not to delay treatment of indeterminate lung lesions $(17,18)$.

Kim et al. reported that lymph node involvement is a risk factor for pulmonary metastatic disease and concluded that, without involvement of the liver or lymph node, a chest CT should not be routinely suggested (13). However, this study demonstrated no correlation between paraaortic lymphadenopathy and lung involvement. According to the data of this research, liver metastasis and a high American joint committee on cancer (AJCC) stage base on the TNM increases the risk of pulmonary metastasis.

In conclusion, although this study was limited by the short-term follow-up, our results suggest that CCT for preoperative staging in CRC patients, especially at stages 3 and 4 in TNM staging and for liver metastases. Further studies to evaluate the effect of CCT findings on long term surveillance and its cost benefit are recommended.

\section{Footnotes}

Authors' Contributions: Study concept and design: Sahar Rabani Khorasgani, Farhad Niaghi, Pooneh Dehghan; acquisition of data: Farhad Niaghi; analysis and interpretation of data: Sahar Rabani Khorasgani, Farhad Niaghi; drafting of the manuscript: Sahar Rabani Khorasgani, Farhad Niaghi; critical revision of the manuscript for important intellectual content: Sahar Rabani Khorasgani, Farhad Niaghi, Pooneh Dehghan; statistical analysis: Sahar Rabani Khorasgani, Farhad Niaghi; administrative, technical, and material support: Sahar Rabani Khorasgani, Farhad Niaghi, Pooneh Dehghan; study supervision: Farhad Niaghi, Pooneh Dehghan

Conflict of Intersts: It is not declared by the authors.

Ethical Approval: This research is approved by Ethical Committee of School of Medicine, Shahid Beheshti University of Medical Sciences (number ID: IR.SBMU.RETECH.REC.1396.420).

Financial Disclosure: The authors have no financial interests related to the material in the manuscript.

Funding/Support: The present article was financially supported by Taleghani Hospital Research Development Committee, School of Medicine, Shahid Beheshti University of Medical Sciences, Tehran, Iran (grand No.: 11211) 


\section{References}

1. Ghahremani R, Yavari P, Khodakarim S, Etemad K, Khosravi A, Ramezani Daryasari R. [The estimated survival rates for colorectal cancer and related factors in Iran from 1384 to 1388 using the aalen's additive risk model]. Iran J Epidemiol. 2016;11:20-9. Persian.

2. Glimelius B, Tiret E, Cervantes A, Arnold D; Esmo Guidelines Working Group. Rectal cancer: ESMO Clinical Practice Guidelines for diagnosis, treatment and follow-up. Ann Oncol. 2013;24 Suppl 6:vi81-8. doi: 10.1093/annonc/mdt240. [PubMed: 24078665].

3. Haaga JR. CT and MRI of the whole body. 6th ed. Philadelphia: Elsevier; 2017.1748 p.

4. Lazzaron AR, Vieira MV, Damin DC. Should preoperative chest computed tomography be performed in all patients with colorectal cancer? Colorectal Dis. 2015;17(10):O184-90. doi: 10.1111/codi.13071. [PubMed: 26201822].

5. Kim HY, Lee SJ, Lee G, Song L, Kim SA, Kim JY, et al. Should preoperative chest CT be recommended to all colon cancer patients? Ann Surg. 2014;259(2):323-8. doi:10.1097/SLA.ob013e3182865080. [PubMed: 23426347].

6. Hogan J, O'Rourke C, Duff G, Burton M, Kelly N, Burke J, et al. Preoperative staging CT thorax in patients with colorectal cancer: Its clinical importance. Dis Colon Rectum. 2014;57(11):1260-6. doi: 10.1097/DCR.0000000000000210. [PubMed: 25285692].

7. Grossmann I, Avenarius JK, Mastboom WJ, Klaase JM. Preoperative staging with chest CT in patients with colorectal carcinoma: Not as a routine procedure. Ann Surg Oncol. 2010;17(8):2045-50. doi: 10.1245/s10434-010-0962-y. [PubMed: 20151212]. [PubMed Central: PMC2899025].

8. Brant WE, Helms CA. Fundamentals of diagnostic radiology. 4th ed. Philadelphia: Lippincott Williams and Wilkins; 2012. p. 22-5.

9. Choi DJ, Kwak JM, Kim J, Woo SU, Kim SH. Preoperative chest computerized tomography in patients with locally advanced mid or lower rectal cancer: Its role in staging and impact on treatment strategy. J Surg Oncol. 2010;102(6):588-92. doi: 10.1002/jso.21651. [PubMed: 20607759].

10. Cho YK, Lee WY, Yi LJ, Park JH, Yun HR, Cho YB, et al. Routine chest computed tomography as a preoperative work-up for primary colorectal cancer: Is there any benefit in short-term outcome? J Korean Surg Soc. 2011;80(5):327-33. doi: 10.4174/jkss.2011.80.5.327. [PubMed: 22066056]. [PubMed Central: PMC3204704].

11. Yongue G, Hotouras A, Murphy J, Mukhtar H, Bhan C, Chan CL. The diagnostic yield of preoperative staging computed tomography of the thorax in colorectal cancer patients without hepatic metastases. Eur J Gastroenterol Hepatol. 2015;27(4):467-70. doi: 10.1097/MEG.0000000000000315. [PubMed: 25874523].

12. Restivo A, Zorcolo L, Piga S, Cocco IM, Casula G. Routine preoperative chest computed tomography does not influence therapeutic strategy in patients with colorectal cancer. Colorectal Dis. 2012;14(5):e21621. doi: 10.1111/j.1463-1318.2012.02878.x. [PubMed: 22469479].

13. Povoski SP, Fong Y, Sgouros SC, Kemeny NE, Downey RJ, Blumgart LH. Role of chest CT in patients with negative chest $\mathrm{x}$-rays referred for hepatic colorectal metastases. Ann Surg Oncol. 1998;5(1):9-15. doi: 10.1007/BF02303757. [PubMed: 9524701].

14. Pavoor RS, Shukla PJ, Milsom JW. The importance of preoperative staging with chest CT scan in patients with colorectal cancer. Ann Surg Oncol. 2011;18 Suppl 3:S224-5. author reply S226-7. doi: 10.1245/s10434010-1306-7. [PubMed: 20839065].

15. Parnaby CN, Bailey W, Balasingam A, Beckert L, Eglinton T, Fife J, et al. Pulmonary staging in colorectal cancer: A review. Colorectal Dis. 2012;14(6):660-70. doi: 10.1111/j.1463-1318.2011.02601.x. [PubMed: 21689294].

16. Brent A, Talbot R, Coyne J, Nash G. Should indeterminate lung lesions reported on staging CT scans influence the management of patients with colorectal cancer? Colorectal Dis. 2007;9(9):816-8. doi: 10.1111/j.1463-1318.2007.01229.x. [PubMed: 17931171].

17. Oh BY, Noh GT, Hong KS, Chung SS, Kim KH, Lee RA. The availability of computed tomography for pulmonary staging in colorectal cancer. Ann Surg Treat Res. 2014;86(4):212-6. doi:10.4174/astr.2014.86.4.212. [PubMed: 24783181]. [PubMed Central: PMC3996719].

18. Ma K, Nayak S, Li H, Evans K, Francescatti A, Brand MI, et al. Radiographic staging practices of newly diagnosed colorectal cancer vary according to medical specialty. Gastrointest Endosc. 2015;82(3):497502. doi:10.1016/j.gie.2015.01.039. [PubMed: 25910667]. 